\title{
O volume de prática em ginástica artística influencia o desempenho das habilidades motoras fundamentais ${ }^{1}$
}

\section{The volume of practice in artistic gymnastics influences the performance of fundamental motor skills}

\section{El volumen de práctica en gimnasia artística influye en el desempeño de las habilidades motoras fundamentales}

(iD) letícia Pimenta Ferreira Escola de Educação Física, Fisioterapia e Terapia Ocupacional da Universidade Federal de Minas Gerais, Belo Horizonte, MG, Brasil. e-mail: leticiapfe@gmail.com

iD Nádia Fernanda Schmitt Marinho Universidade Salgado de Oliveira, Belo Horizonte, MG, Brasil e-mail: nfsmarinho@gmail.com

(iD) Patrick Costa Ribeiro-Silva Fundação de Ensino e Pesquisa do Sul de Minas- Centro Universitário do Sul de Minas, Centro de Saúde, Varginha, Brasil. e-mail: patrick.gedam35@gmail.com

(iD) Welisney Soares de Brito Escola de Educação Física, Fisioterapia e Terapia Ocupacional da Universidade Federal de Minas Gerais, Belo Horizonte, MG, Brasil e-mail: welisneymodas@hotmail.com

iD Rodolfo Novellino Benda Escola Superior de Educação Física, Universidade Federal de Pelotas, Pelotas, RS, Brasil e-mail: rodolfobenda@yahoo.com.br

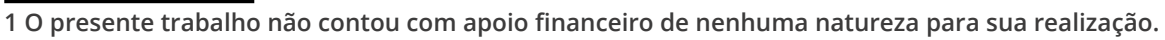


Resumo: O objetivo do presente estudo foi investigar o volume de prática em ginástica artística no desempenho de habilidades motoras fundamentais. Para tal, 22 ginastas (idade média=8,9 $\pm 1,54$ ) foram divididas em grupo escola de esportes e grupo equipe. A avaliação do desempenho motor foi realizada pelo TGMD-2. Os resultados, analisados pelos testes de Mann-Whitney e a análise qui-quadrado, indicaram desempenho superior do grupo equipe em habilidades fundamentais locomotoras, bem como no quociente motor. Conclui-se que o volume de prática de ginástica artística pode beneficiar o desempenho de habilidades fundamentais locomotoras, mas não de controle de objetos. Assim, parece haver uma relação de especificidade quanto às categorias de habilidades praticadas na ginástica artística versus habilidades fundamentais desenvolvidas.

Palavras-chave: Desenvolvimento motor. Habilidades motoras fundamentais. Iniciação esportiva. Ginástica Artística.

Abstract: The aim of the present study was to investigate the volume of practice in artistic gymnastics on the motor performance of fundamental skills. For this, 22 gymnasts (mean age $=8.9 \pm 1.54$ ) were divided into a sports school group and a team group. The assessment of motor performance was performed by TGMD-2. The results, analyzed by the Mann-Whitney tests and the chi-square analysis, indicated superior performance of the team group in fundamental locomotor skills, as well as in the motor quotient. As conclusion, the volume of practice in artistic gymnastics may benefit the performance of locomotor fundamental skills, but not object control skills. Thus, there seems to be a relationship of specificity regarding the categories of skills practiced in artistic gymnastics versus fundamental skills developed.

Keywords: Motor development. Fundamental skills. Sports introduction. Artistic Gymnastic.

Resumen: El objetivo del presente estudio fue investigar el volumen de práctica en gimnasia artística sobre el rendimiento motor de habilidades fundamentales. Para ello, 22 gimnastas (edad media = 8,9 
$\pm 1,54$ ) se dividieron en un grupo de escuela deportiva y un grupo de equipo. La evaluación del rendimiento motor fue realizada por TGMD2. Se concluye que el volumen de práctica de gimnasia artística puede beneficiar el desempeño de las habilidades locomotoras fundamentales, pero no el control de objetos. Por lo tanto, parece haber una relación de especificidad con respecto a las categorías de habilidades practicadas en gimnasia artística versus habilidades fundamentales desarrolladas.

Palabras clave: Desarrollo motor. Habilidades fundamentales. Iniciación deportiva. Gimnasia artística.

Submetido em: 10-03-2021

Aceito em: 13-04-2021 
O volume de prática em ginástica artística influencia o desempenho das habilidades... letícia Pimenta Ferreira • Nádia Fernanda Schmitt Marinho • Patrick Costa Ribeiro-Silva • Welisney Soares de Brito $\cdot$ Rodolfo Novellino Benda

\section{Introdução}

O desenvolvimento motor é um processo contínuo de mudanças no comportamento motor ao longo do ciclo de vida (GALLAHUE; OZMUN; GOODWAY, 2013), inferido em estudos da área pelo desempenho em habilidades motoras em diferentes momentos do processo. Tais mudanças foram inicialmente sistematizadas na categorização de movimentos predominantemente observados na população em cada faixa etária e vinculados a fases de desenvolvimento motor. Vários modelos descritivos foram propostos, com uma lógica sequencial, sendo o período entre o nascimento e o início da adolescência aquele que apresenta maior número de fases de desenvolvimento motor (GALLAHUE, 1982; HAUBENSTRICKER; SEEFELDT, 1986; TANI; MANOEL; KOKUBUN; PROENÇA, 1988; GALLAHUE, 1989). Dentre os modelos descritivos, destaca-se a Ampulheta, que foi apresentada em 1989 (GALLAHUE, 1989), cuja proposta é referenciada até os dias atuais (GALLAHUE; OZMUN; GOODWAY, 2013; SAMPAIO; VALENTINI, 2015; SANTOS; PACHECO; BASSO; TANI, 2016; BARDID; HUYBEN; LENOIR; SEGHERS; MARTELAER; GOODWAY, 2016).

O processo de desenvolvimento motor foi inicialmente creditado apenas a componentes genéticos e maturacionais (GESELL, 1929; GESELL; THOMPSON, 1929), pautados em um paradigma pré-formacionista (AUSUBEL,1958) que vigorou até a década de 1970. A partir desse momento, uma abordagem dinâmica passou a ser predominante (TANI; MEIRA JÚNIOR; UGRINOWITSCH; BENDA; CHIVIACOWSKY; CORRÊA, 2010; GALLAHUE; OZMUN; GOODWAY, 2013; BASSO; SANTOS, BENDA, 2016), a qual preconiza que um comportamento motor emergente é produto da interação entre diversos fatores, nomeadamente a pessoa, a tarefa e o contexto ambiental em que está inserido (NEWELL, 1986). Esta visão assume que alterações nas características da pessoa, no objetivo da tarefa ou no contexto do ambiente poderão levar a comportamentos distintos daqueles apresentados anteriormente. Por exemplo, 
O volume de prática em ginástica artística influencia o desempenho das habilidades... letícia Pimenta Ferreira • Nádia Fernanda Schmitt Marinho • Patrick Costa Ribeiro-Silva • Welisney Soares de Brito $\cdot$ Rodolfo Novellino Benda

observou-se diferenças nos comportamentos reflexivos à medida que se verificou alterações de peso de bebês ao longo de 4 meses, ou mesmo conforme o ambiente em que os bebês foram posicionados (dentro ou fora da água) (THELEN; FISHER; RIDLEYJOHNSON, 1984).

Portanto, conforme a concepção dinâmica preconiza, mudanças no contexto ambiental podem levar a comportamentos emergentes distintos (OYAMA, 2009). Assim, assume-se que fatores ambientais, relacionados à cultura, à qualidade de prática ou à quantidade de experiência podem influenciar no processo de desenvolvimento motor, na aquisição de habilidades fundamentais (BARDID; HUYBEN; LENOIR; SEGHERS; MARTELAER; GOODWAY, 2016; SANTOS; PACHECO; BASSO; TANI, 2016). Dentre atividades cotidianas do contexto sociocultural de crianças e adolescentes, a prática esportiva fora do ambiente escolar pode ser apontada como um fator presente, considerando que há oferta para sua prática em diferentes instituições e com diferentes objetivos (QUEIROZ; RÉ; HENRIQUE; MOURA; CATTUZZO, 2014). Estudos têm verificado que a oferta de prática de esportes sistematizada para crianças contribui para o desempenho de habilidades motoras fundamentais (LUCCA; KREBS; RAMALHO; SANTOS; NOBRE; TRICHES, 2010; NAZÁRIO; VIEIRA, 2014; QUEIROZ; RÉ; HENRIQUE; MOURA; CATTUZZO, 2014; RIBEIRO-SILVA; MARINHO; BRITO; COSTA; BENDA, 2018). É importante destacar que, mesmo sem uma análise mais aprofundada do conteúdo aplicado nessas práticas esportivas, não está claro se a prática de habilidades motoras especializadas, ou seja, os fundamentos técnicos das modalidades envolvidas, contribui para o desempenho de habilidades motoras fundamentais. Assumir essa conclusão significaria a inversão do caminho proposto nas tradicionais sequências de desenvolvimento motor (GALLAHUE, 1982; HAUBENSTRICKER; SEEFELDT, 1986; TANI; MANOEL; KOKUBUN; PROENÇA, 1988; GALLAHUE, 1989).

Apesar de os estudos terem investigado o desempenho motor em habilidades fundamentais de crianças participantes de diferentes práticas esportivas (BASTIK; KALKAVAN; YAMANER; SAHIN; 
O volume de prática em ginástica artística influencia o desempenho das habilidades... letícia Pimenta Ferreira • Nádia Fernanda Schmitt Marinho • Patrick Costa Ribeiro-Silva • Welisney Soares de Brito $\cdot$ Rodolfo Novellino Benda

GULLU, 2012; NAZÁRIO; VIEIRA, 2014; RIBEIRO-SILVA; MARINHO; BRITO; COSTA; BENDA, 2018), ainda não foi investigado qual a influência do nível de rendimento em uma mesma modalidade esportiva no desempenho de tais habilidades. Por exemplo, a prática de esportes pode visar a introdução à modalidade, sendo ofertada de modo a ampliar as experiências motoras da criança, como também há casos de crianças serem submetidas à prática esportiva com vistas a alcançar alto rendimento para participação em competições de âmbito nacional. Em outras palavras, há diferentes modos de se praticar uma mesma modalidade esportiva.

O esporte é um fenômeno sociocultural cujas manifestações são classificadas em esporte-educação, esporte-participação (lazer) e esporte-performance (KRAVCHYCHYN; LIMA; OLIVEIRA; BARBOSA-RINALDI; LARA, 2012). Esporte-performance visa à competição e para isso o volume de prática deve ser maior para o treinamento minucioso das técnicas da modalidade. Por outro lado, o esporte-educação visa à formação do cidadão e, nesse sentido, o conteúdo das aulas de esporte busca a experimentação motora, visando à exploração de ações corporais, em sintonia com o nível de desenvolvimento motor esperado para a idade, evitando-se a especialização esportiva precoce (KREBS, 1992; TANI, 2001; GRECO; BENDA, 2006; PAES, 2006; GRECO; MORALES; ABURACHID; LÓPEZ; SILVA; BENDA, 2015). Quando a prática esportiva privilegia a ampliação de experiências motoras e, consequentemente, o seu repertório motor, espera-se melhor desempenho das habilidades fundamentais; ao passo que quando a prática esportiva focaliza no desempenho da técnica esportiva, há a expectativa que os fundamentos técnicos da modalidade sejam aprendidos em detrimento das habilidades fundamentais, prejudicando a fase de desenvolvimento na qual a criança se encontra (TANI, 2001).

Assim, o objetivo do presente estudo foi investigar a influência do volume de prática em ginástica artística no desempenho de habilidades motoras fundamentais. A escolha da modalidade se deveu ao fato de tradicionalmente se observar, ao mesmo tempo, crianças em seus primeiros contatos com o esporte e também 
O volume de prática em ginástica artística influencia o desempenho das habilidades... letícia Pimenta Ferreira • Nádia Fernanda Schmitt Marinho • Patrick Costa Ribeiro-Silva • Welisney Soares de Brito $\cdot$ Rodolfo Novellino Benda

crianças com as mesmas faixas etárias com desempenhos de excelência, o que permitiu a análise. Para tanto, a seguinte hipótese foi estabelecida: crianças com menor volume de prática em Ginástica Artística (Iniciação) apresentam desempenho motor superior em habilidades fundamentais do que crianças com maior volume de prática em Ginástica Artística (Equipe).

\section{Procedimentos metodológicos}

\section{População e Amostra}

O estudo foi realizado com 22 voluntárias do sexo feminino praticantes da modalidade de Ginástica Artística de uma instituição de ensino de Minas Gerais. A faixa etária foi de 6 a 10 anos de idade (idade média de 8,9 $\pm 1,54$ anos). $O$ estudo foi aprovado pelo Comitê de Ética em Pesquisa da Universidade Federal de Minas Gerais sob o número de Parecer CAAE - 16380613.8.0000.5149.

\section{Instrumento}

Para avaliação do desempenho motor, foi utilizado o Test of Gross Motor Development (TGMD-2) (ULRICH, 2000), composto por 12 itens, dividido em dois sub-testes: habilidades locomotoras e habilidades de controle de objetos. Seis habilidades locomotoras (correr, galopar, salto sobre o mesmo pé, salto horizontal, salto sobre obstáculo e corrida lateral) e seis habilidades de controle de objetos (rebater com bastão, quicar, receber, chutar, arremessar e rolar a bola) foram avaliadas. Destaque-se que, destas 12 habilidades fundamentais avaliadas no teste (locomotoras e controle de objetos), apenas correr, salto horizontal e salto sobre obstáculo são usuais na prática de ginástica artísticas. As demais nove habilidades fundamentais não são comuns no treinamento da modalidade. 
O volume de prática em ginástica artística influencia o desempenho das habilidades... letícia Pimenta Ferreira • Nádia Fernanda Schmitt Marinho • Patrick Costa Ribeiro-Silva • Welisney Soares de Brito $\cdot$ Rodolfo Novellino Benda

A aplicação do teste levou aproximadamente 20 minutos por criança. Os dados do desempenho motor das crianças foram analisados a partir do registro em duas câmeras filmadoras. O desempenho da criança em cada habilidade foi avaliado com 1 ponto quando o padrão executado correspondeu ao critério estabelecido no teste. Quando o critério não foi atendido, a pontuação foi zero. A partir dos escores brutos do desempenho nos subtestes (locomotor e controle de objetos) e da idade cronológica da criança é identificado um standard escore. Este escore permite classificar a criança em categorias descritivas divididas em: muito superior, superior, acima da média, média, abaixo da média, pobre e muito pobre. O teste ainda fornece um Quociente Motor Global, composto pelo resultado da soma do standard escore dos dois subtestes, sendo o teste validado para o idioma português (VALENTINI, 2012).

\section{Delineamento}

Dois grupos foram formados $(n=11)$ com idades pareadas e de acordo com o nível de rendimento na modalidade esportiva Ginástica Artística (GA): Grupo Equipe e Grupo Escola de Esportes. As crianças da equipe de treinamento foram convidadas a participar da pesquisa como parte do Grupo Equipe. Sua rotina de treinamento abrangia de 18 a $20 \mathrm{~h}$ de treino semanal e participação em competições nacionais e estaduais após completar 8 anos de idade. As crianças da escolinha foram convidadas a participar e formaram o Grupo Escola de Esportes. Sua rotina de treinamento abrangia 2 a $4 \mathrm{~h}$ semanais e não participavam de competições. Os grupos foram pareados por idade e cada um foi constituído por duas crianças de 6 anos, uma criança de 8 anos, duas com 9 anos e seis com 10 anos.

\section{Procedimentos}

Primeiramente, os pais das crianças foram contatados e forneceram o consentimento para que suas filhas participassem do estudo. Após a autorização, foi agendada e então realizada a 
O volume de prática em ginástica artística influencia o desempenho das habilidades... letícia Pimenta Ferreira • Nádia Fernanda Schmitt Marinho • Patrick Costa Ribeiro-Silva • Welisney Soares de Brito $\cdot$ Rodolfo Novellino Benda

avaliação do desempenho motor individualmente, em que a execução de cada habilidade motora fundamental se deu em duas tentativas. Toda a aplicação do teste foi filmada com uma câmera frontal e outra lateral. Conforme o protocolo do teste, o avaliador demonstrou cada habilidade, certificando-se de que os avaliados compreenderam o que deveriam executar. Os testes foram analisados através dos vídeos gravados por dois avaliadores. A consistência entre observadores apontou índices aceitáveis, tanto para habilidades locomotoras $(0,982)$ quanto para habilidades de controle de objeto $(0,988)$.

\section{Análise Estatística}

Para análise do escore bruto de Habilidades Locomotoras, de Controle de Objetos, e do Quociente Motor Global foi utilizado o teste não paramétrico de Mann-Whitney. A análise dos dados descritivos foi realizada por distribuição de frequência, utilizando o teste Qui-quadrado para verificar a associação entre o quociente motor global e o nível de rendimento em ginástica artística. O nível de significância estatístico adotado foi de $p \leq 0,05$.

\section{Resultados}

Inicialmente serão apresentados os resultados da média do desempenho nos subtestes Locomotor e de Controle de Objetos a partir do escore bruto. Posteriormente serão apresentados os dados do Quociente Motor e, por fim, os dados da análise descritiva.

Os valores de escore bruto Locomotor (Gráfico 1) encontrado para o grupo Equipe foi, conforme o teste Mann-Whitney, significantemente superior ao encontrado para o grupo Escola de Esportes $[Z(N=22)=3,12, p=0,0018]$. Em relação ao escore bruto de Controle de Objetos (Gráfico 1), o teste de Mann-Whitney não revelou diferença significante entre os grupos Equipe e Escola de Esportes $[Z(N=22)=1,54, p=0,123]$. 
O volume de prática em ginástica artística influencia o desempenho das habilidades... letícia Pimenta Ferreira • Nádia Fernanda Schmitt Marinho • Patrick Costa Ribeiro-Silva • Welisney Soares de Brito $\cdot$ Rodolfo Novellino Benda

Gráfico 1. Escore bruto do desempenho em habilidades locomotoras e de controle de objetos das crianças do Grupo Equipe e do Grupo Escola de Esportes.

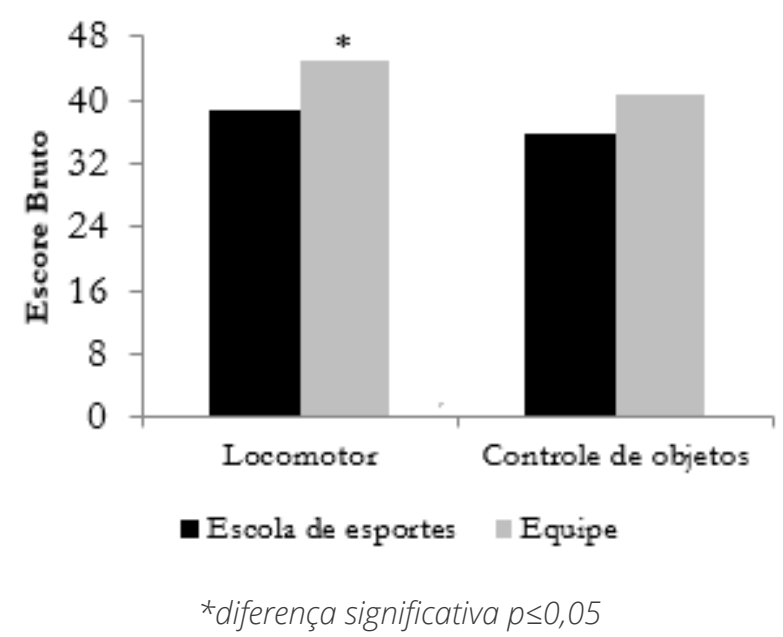

Para o Quociente Motor (Gráfico 2), o teste Mann-Whitney mostrou superioridade significante do grupo Equipe (*) sobre 0 grupo Escola de Esportes [ $(\mathrm{N}=22)=3,18, \mathrm{p}=0,0014]$.

Gráfico 2. Quociente Motor das e crianças do Grupo Equipe e do Grupo Escola de Esportes

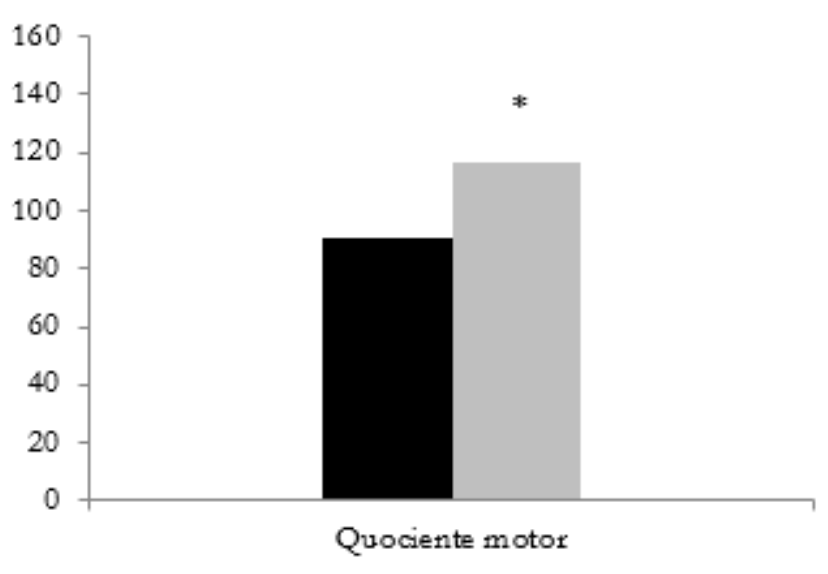

Escola de esportes Equipe

*diferença significativa $p \leq 0,05$ 
O volume de prática em ginástica artística influencia o desempenho das habilidades... letícia Pimenta Ferreira • Nádia Fernanda Schmitt Marinho • Patrick Costa Ribeiro-Silva • Welisney Soares de Brito $\cdot$ Rodolfo Novellino Benda

A análise qualitativa do TGMD-2 indicou, por meio do teste Quiquadrado, uma associação significante entre o quociente motor global e o nível de rendimento em Ginástica Artística (X2=6,349, p= 0,042). As participantes do grupo Equipe apresentaram associação positiva com os níveis superior e muito superior de desempenho motor $(* *)$. Por outro lado, as participantes do grupo Escola de Esportes apresentaram associação positiva com os níveis pobre e abaixo da média (*) de desempenho motor (Gráfico 3).

Gráfico 3. Análise de categorias de crianças do grupo Equipe e grupo Escola de Esportes.

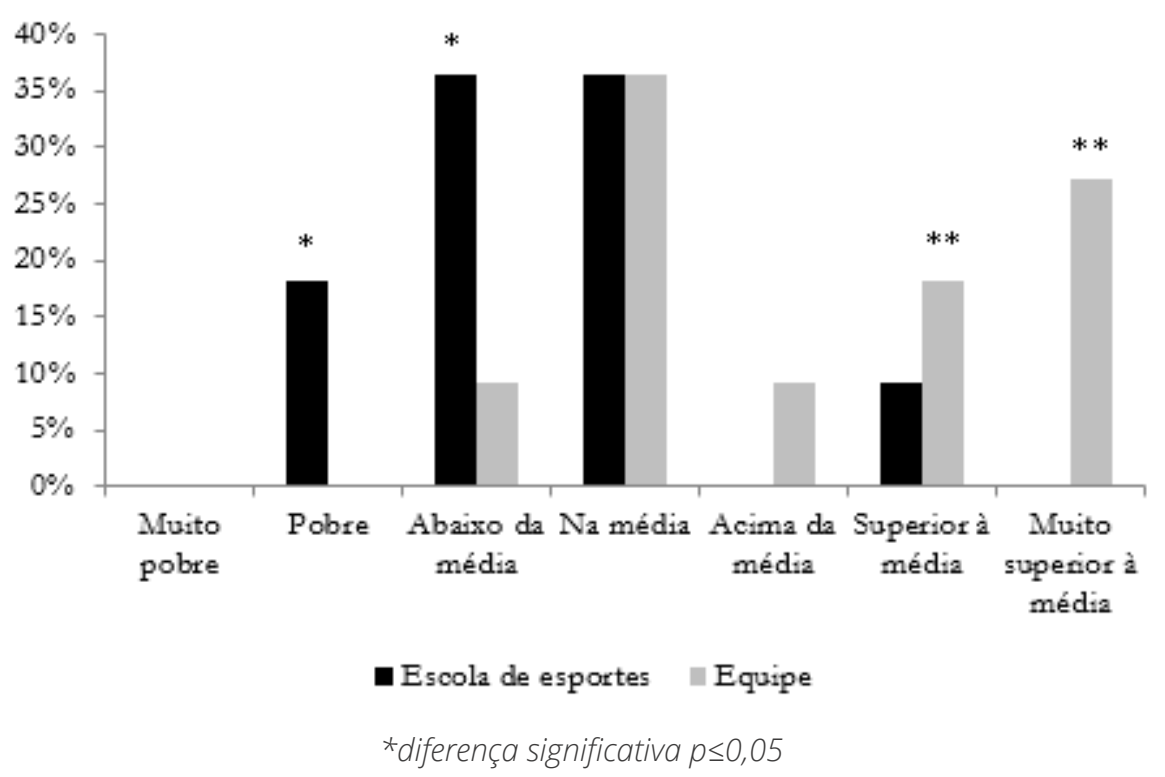

\section{Discussão}

O presente estudo teve como objetivo investigar a influência do volume de prática em ginástica artística no desempenho de habilidades motoras fundamentais. A hipótese formulada foi de que crianças com menor volume de prática em Ginástica Artística (Iniciação) apresentam desempenho motor superior em habilidades fundamentais do que crianças com maior volume de prática em Ginástica Artística (Equipe). O estabelecimento dessa hipótese baseia-se na premissa de que a prática esportiva visando ao alto 
O volume de prática em ginástica artística influencia o desempenho das habilidades... letícia Pimenta Ferreira • Nádia Fernanda Schmitt Marinho • Patrick Costa Ribeiro-Silva • Welisney Soares de Brito $\cdot$ Rodolfo Novellino Benda

rendimento exige maior volume de prática dedicado ao aperfeiçoamento dos fundamentos técnicos, no presente estudo, o caso das atletas da Equipe. Por outro lado, as crianças participantes do Grupo Escola de Esportes tinham menor volume de prática em Ginástica Artística, permitindo mais tempo disponível para a prática de atividades motoras livres, que podem privilegiar o aumento e diversificação do repertório motor por meio da prática de habilidades fundamentais, jogos e brincadeiras populares, além da prática de diferentes modalidades esportivas visando à aprendizagem ou lazer (KREBS, 1992; TANI, 2001; GRECO; BENDA, 2006; PAES, 2006; GRECO; MORALES; ABURACHID; LÓPEZ; SILVA; BENDA, 2015).

Os resultados encontrados apontaram que a maioria das crianças praticantes de Ginástica Artística em Iniciação não apresentou nível de desempenho motor de habilidades fundamentais esperado para suas idades correspondentes. Os resultados ainda mostraram que atletas de Ginástica Artística pertencentes à equipe de competição apresentaram nível de desempenho motor de habilidades fundamentais adequadas para suas idades correspondentes. Ainda, o grupo da Equipe teve desempenho superior ao grupo de Iniciação nas habilidades de Locomoção e no Quociente Motor Global. Tais resultados rejeitam a hipótese do estudo e sugerem uma possível explicação alternativa, pois mostram a superioridade do grupo que tem maior volume de prática em Ginástica Artística, ou seja, uma prática especializada na modalidade. Os resultados também demonstraram similaridade entre os grupos no desempenho de habilidades de controle de objetos, o que era de certa forma esperado, uma vez que a modalidade não fomenta este tipo de habilidade, corroborando a ideia de que experiências específicas levam a cursos de desenvolvimento específicos (GOTTLIEB, 1992).

De modo geral, assume-se que o nível de rendimento na Ginástica Artística influenciou o desempenho nas habilidades motoras fundamentais de forma inversa daquela esperada. A superioridade do grupo Equipe de Ginástica Artística no desempenho motor de habilidades locomotoras e Quociente Motor refutou a 
O volume de prática em ginástica artística influencia o desempenho das habilidades... letícia Pimenta Ferreira • Nádia Fernanda Schmitt Marinho • Patrick Costa Ribeiro-Silva • Welisney Soares de Brito $\cdot$ Rodolfo Novellino Benda

hipótese que indicava comportamento superior do grupo Escola de Esportes. Em outras palavras, os resultados mostraram-se contrários à ideia de que a prática especializada visando ao alto rendimento na modalidade levaria à especialização em fundamentos técnicos e restringiria uma prática motora generalizada, responsável pelo desenvolvimento de habilidades fundamentais (GALLAHUE; 1982; TANI; MANOEL; KOKUBUN; PROENÇA, 1988; TANI, 2001; GALLAHUE; OZMUN; GOODWAY, 2013).

A Metáfora da Montanha (CLARK; METCALFE, 2002) sugere que, para subir a montanha das habilidades especializadas, o ser humano desce à base da montanha relacionada às habilidades fundamentais que precisa para, a partir dela, subir ao cume. Os resultados do presente estudo permitem inferir que existe uma relação bidirecional ao longo do processo de mudança. Não apenas o ser humano desce à base fundamental para poder subir ao cume da habilidade especializada, como o processo de subir ao cume parece também contribuir para tornar a base aperfeiçoada. Em outras palavras, é possível especular que a prática de modalidades esportivas por crianças contribui para o desempenho em habilidades fundamentais (PÍFFERO; VALENTINI, 2010; RIBEIROSILVA; MARINHO; BRITO; COSTA; BENDA, 2018).

Outra possível explicação remete ao contexto observado em uma série de estudos recentes que apontam para atrasos motores em crianças da mesma faixa etária (PALMA; CAMARGO; PONTES, 2012; RÉ; LOGAN; CATTUZZO; HENRIQUE; TUDELA; STODDEN, 2018; SANTOS; SILVA; VILLANUEVA; SILVA JÚNIOR; CATTUZZO; RÉ, 2020). O diagnóstico de atraso motor tem sido associado a um contexto em que as atividades de lazer sedentário são preferidas pelas crianças (STABELINI NETO; MASCARENHAS; NUNES; LEPRE; CAMPOS, 2004; RÉ; LOGAN; CATTUZZO; HENRIQUE; TUDELA; STODDEN, 2018). Nesse sentido, a prática de atividade física sistemática, por mais horas, pode ter agido como estímulo necessário ao desenvolvimento motor (TANI, 1987; TANI; MANOEL; KOKUBUN; PROENÇA, 1988; PÍFFERO; VALENTINI, 2010; GALLAHUE; OZMUN; GOODWAY, 2013). Assim sendo, a questão passaria a ser a quantidade de prática e não apenas o tipo de atividade praticada. 
O volume de prática em ginástica artística influencia o desempenho das habilidades... letícia Pimenta Ferreira • Nádia Fernanda Schmitt Marinho • Patrick Costa Ribeiro-Silva • Welisney Soares de Brito $\cdot$ Rodolfo Novellino Benda

É ainda possível especular que a explicação para os resultados seja anterior à própria prática da ginástica, tanto em menor quanto em maior volume de prática. Talvez a seleção das crianças que foram convidadas a participar da equipe de competição tenha considerado o desempenho da criança em habilidades fundamentais. Em outras palavras, teriam sido selecionadas aquelas crianças com melhor nível de desempenho nas habilidades cotidianas, dentre elas as habilidades fundamentais. Isto é, aquelas crianças que apresentariam melhor desempenho motor foram escolhidas para participar da equipe de competição. Obviamente, este argumento merece ser testado em estudos posteriores.

O estudo apresenta ainda algumas limitações. Em primeiro lugar, trata-se de uma amostra reduzida, o que é insuficiente para uma generalização mais ampliada do fenômeno. Porém, não foi fácil encontrar uma amostra maior, especialmente porque envolveu crianças de até 12 anos praticantes de Ginástica Artística em uma carga horária semanal superior a 15 horas, além da participação em competições em nível estadual. Segundo, após a análise dos resultados e redação da discussão, verificou-se que teria sido muito importante ter mais dados sobre que tipo de atividades estas crianças fazem em seus cotidianos quando estão nas suas residências, nas escolas, isto é, fora do horário de prática esportiva. Tais informações poderiam ter contribuído para explicar os dados obtidos.

Em síntese, os resultados do presente estudo indicam que o maior volume de prática em uma modalidade esportiva pode ter influência positiva sobre o desempenho motor em habilidades fundamentais de crianças. Tal influência parece ser direcionada pela quantidade de prática semanal de atividade motora. É possível também verificar, pela não diferença entre os grupos nas habilidades de controle de objeto, que essa possível influência se dá mediante a especificidade da prática. Sugere-se que novos estudos relacionando especialização precoce e desenvolvimento das habilidades motoras fundamentais sejam desenvolvidos, inclusive com outras modalidades esportivas. Estudos futuros poderiam 
O volume de prática em ginástica artística influencia o desempenho das habilidades... letícia Pimenta Ferreira • Nádia Fernanda Schmitt Marinho • Patrick Costa Ribeiro-Silva • Welisney Soares de Brito $\cdot$ Rodolfo Novellino Benda

analisar crianças praticantes de modalidades em que controle de objetos é predominante e verificar possíveis efeitos no desempenho de habilidades locomotoras, de acordo com a especificidade da prática. Ainda, estudos que investiguem os critérios utilizados para a seleção de crianças para participar de equipes de competição podem esclarecer se o desempenho motor em habilidades fundamentais contribui para tal escolha. Além disso, o uso de instrumentos para avaliar as atividades que as crianças realizam no seu cotidiano parece ser essencial para explicar o desempenho de habilidades fundamentais.

\title{
Referências
}

\author{
AUSUBEL, D. P. Theory and problems of child development. \\ New York: Grune \& Stratton; 1958. \\ BARDID, F.; HUYBEN, F.; LENOIR, M.; SEGHERS, J.; MARTELAER, K. \\ D.; GOODWAY, J. D. Assessing fundamental motor skills in Belgian \\ children aged 3-8 years highlights differences to US reference \\ sample. Acta Paediatr, [s. I.], v. 105, n. 6, p. 281-290, 2016. \\ BASSO, L.; SANTOS, F. G.; BENDA, R. N. Estudo do desen- \\ volvimento motor: tendências e perspectivas. In: TANI, G.
}

Comportamento motor: conceitos, estudos e aplicações. Rio de Janeiro: Guanabara-Koogan; 2016. p. 25-30.

BASTIK, C.; KALKAVAN, A.; YAMANER, F.; SAHIN, S.; GULLU, A. Investigation of basic motor skills according to TGMD-2 test on male athletes of 10 ages group who participated to competitions in different sports branches. Procedia Soc Behav Sci, [s. I.], v. 46, p. 4741-4745, 2012.

CLARK J. E.; METCALFE, J. M. The mountain of motor development: A metaphor. In: CLARK, J. E.; HUMPHREY, J. H. (eds.) Motor development: Research and reviews. Reston, VA: NASPE Publications; 2002. p.163-190. 
O volume de prática em ginástica artística influencia o desempenho das habilidades... letícia Pimenta Ferreira • Nádia Fernanda Schmitt Marinho • Patrick Costa Ribeiro-Silva • Welisney Soares de Brito $\cdot$ Rodolfo Novellino Benda

GALLAHUE, D. Understanding motor development in children. Nova York: John Wiley e Sons; 1982.

GALLAHUE, D. Understanding motor development: infants, children, and adolescents. 2. ed. Indianapolis: Benchmark Press; 1989.

GALLAHUE, D. L.; OZMUN, J. C.; GOODWAY J. D. Compreendendo o desenvolvimento motor: bebês, crianças, adolescentes e adultos. 3. ed. São Paulo: Phorte, 2013.

GESELL, A. Maturation and infant behavior pattern. Psychol Rev., Washington, v. 36, n. 4, p. 307-319, 1929.

GESELL, A.; THOMPSON, H. Learning and growth in identical infant twins. Genet. Psychol. Monogr., Massachussets, v. 6, n. 1, p. 3-124, 1929.

GOTTLIEB, G. Individual development and evolution: The genesis of novel behavior. New York: Oxford University Press, 1992.

GRECO, P. J.; BENDA, R. N. Iniciação aos esportes coletivos: uma escola da bola para crianças e adolescentes. In: ROSE, D. J. (ed.). Modalidades esportivas coletivas, Rio de Janeiro: Guanabara Koogan, 2006. p. 180-193.

GRECO, P. J.; MORALES, J. C. P.; ABURACHID, L. C.; LÓPEZ, M. C.; SILVA, S. R.; BENDA, R. N. Iniciação esportiva universal: o jogo do "ABC" na alfabetização esportiva. In: LEMOS, K. L. M.; GRECO, P. J.; MORALES, J. C. P. 5 Congresso Internacional dos Jogos Desportivos. Belo Horizonte: Instituto Casa da Educação Física; 2015. p. 335-359.

HAUBENSTRICKER, J.; SEEFELDT, V. Acquisition of motor skills during childhood. In: SEEFELDT, V. (ed.), Physical activity \& well-being. Reston: American Alliance for Health, Physical Education, Recreation, and Dance; 1986. p. 41-102.

KREBS, R. J. Da estimulação à especialização: primeiro esboço de uma teoria da especialização motora. Kinesis, Santa Maria, v. 9, n. 9, p. 29-44, 1992. 
O volume de prática em ginástica artística influencia o desempenho das habilidades... letícia Pimenta Ferreira • Nádia Fernanda Schmitt Marinho • Patrick Costa Ribeiro-Silva • Welisney Soares de Brito $\cdot$ Rodolfo Novellino Benda

KRAVCHYCHYN, C.; LIMA, W. F.; OLIVEIRA, A. A. B.; BARBOSARINALDI, I. P.; LARA, L. M. Estudos brasileiros sobre o esporte: ênfase no esporte-educação. Movimento, Porto Alegre, v. 18, n. 2, p. 339-350, 2012.

LUCCA, M. D.; KREBS, R. J.; RAMALHO, M. H. S.; SANTOS, J. O. L.; NOBRE, G. C.; TRICHES, J. R. A contribuição da prática do handebol no desempenho das habilidades motoras amplas de escolares. Cinergis, Santa Cruz do Sul, v. 11, n. 9, p. 1-8, 2010. NAZÁRIO, P. F.; VIEIRA, J. L. L. Sport context and the motor development of children. Rev. Bras. Cineantropom. Desempenho Hum., Florianópolis, v. 16, n. 1, p. 86-95, 2014.

NEWELL, K. M. Constraints on the development of coordination. In: WADE, M.G, WHITING, H.T.A. (eds.) Motor development in children: Aspects of coordination and control. Boston: Martin Nighoff; 1986. p. 341-60.

OYAMA, S. Friends, neighbors, and boundaries. Ecol. Psychol., [s. I.], v. 21, n. 2, p. 147-54, 2009.

PAES, R. R. Pedagogia do esporte: especialização esportiva precoce. In: TANI, G., BENTO, J. O.; PETERSEN, R. D. S. Pedagogia do desporto. Rio de Janeiro: Guanabara Koogan; 2006. p. 219-26. PALMA, M. S.; CAMARGO, V. A.; PONTES, M. F. P. Efeitos da atividade física sistemática sobre o desempenho motor de crianças pré-escolares. Rev. Educ. Fís./UEM, Maringá, v. 23, n. 3, p. 421 429, 2012.

PÍFFERO, C. M.; VALENTINI, N. C. Habilidades especializadas do tênis: um estudo de intervenção na iniciação esportiva com crianças escolares. Rev. Bras. Educ. Fís., São Paulo, v. 24, p.149-163, 2010.

QUEIROZ, D. R.; RÉ, A. H. N.; HENRIQUE, R. S.; MOURA, M. S.; CATTUZZO, M. T. Participation in sports practice and motor competence in preschoolers. Motriz Rev. Educ. Fís., Rio Claro, v. 20, n. 1, p.26-32, 2014. 
O volume de prática em ginástica artística influencia o desempenho das habilidades... letícia Pimenta Ferreira • Nádia Fernanda Schmitt Marinho • Patrick Costa Ribeiro-Silva • Welisney Soares de Brito $\cdot$ Rodolfo Novellino Benda

RÉ, A. H. N.; LOGAN, S. W.; CATTUZZO, M. T.; HENRIQUE, R. D. S.; TUDELA, M. C.; STODDEN, D. F. Comparison of motor competence levels on two assessments across childhood. J. Sports Sci., [s. I.], v. 36, n. 1, p. 1-6, 2018.

RIBEIRO-SILVA, P. C.; MARINHO, N. F. S.; BRITO, W. S.; COSTA, N. E.; BENDA, R. N. Desempenho motor em habilidades básicas de crianças participantes e não participantes de prática esportiva orientada. Rev. Educ. Fís./UEM, Maringá, v. 29, e2903, 2018.

SAMPAIO, D. F.; VALENTINI, N. F. Iniciação esportiva em ginástica rítmica: abordagens tradicional e o clima motivacional para a maestria. Rev. Educ. Fís./UEM, Maringá, v. 26, n. 1, p.1-10, 2015. SANTOS, F. G.; PACHECO, M. M.; BASSO, L.; TANI, G. A comparative study of the mastery of fundamental movement skills between different cultures. Motricidade, Ribeira de Pena, v. 12, n. 2, p. 116-26, 2016.

SANTOS, G.; SILVA, M. M. L. M.; VILLANUEVA, M. D.; SILVA JÚNIOR, J. P.; CATTUZZO, M. T.; RÉ, A. H. N. Competência motora de crianças pré-escolares brasileiras avaliadas pelo teste TGMD-2: uma revisão sistemática. Journal of Physical Education, Maringá, v. 31, e3117, 2020.

STABELINI NETO, A.; MASCARENHAS, L. P. G.; NUNES, G. F.; LEPRE, C.; CAMPOS, W. Relação entre fatores ambientais e habilidades motoras básicas em crianças de 6 e 7 anos. Revista Mackenzie de Educação Física e Esporte, São Paulo, v. 3, n. 3, p. 135-140, 2004.

TANI, G. Educação Física na Pré-Escola e nas Quatro Primeiras Séries do Ensino de Primeiro Grau: Uma Abordagem de Desenvolvimento. Kinesis, Santa Maria, v. 3, n. 1, p. 19-41, 1987.

TANI, G. A criança no esporte: implicações da iniciação esportiva precoce. In: KREBS, R. J.; COPETTI, F.; ROSO, M. R.; KROEFF, M. S.; SOUZA, $P$. H. (eds.). Desenvolvimento infantil em contexto. Florianópolis: UDESC, 2001. p. 213-24.

TANI, G., MANOEL, E. J.; KOKUBUN, E.; PROENÇA, J. E. Educação física escolar. São Paulo: EPU/Edusp; 1988. 
O volume de prática em ginástica artística influencia o desempenho das habilidades... letícia Pimenta Ferreira • Nádia Fernanda Schmitt Marinho • Patrick Costa Ribeiro-Silva • Welisney Soares de Brito $\cdot$ Rodolfo Novellino Benda

TANI G.; MEIRA JÚNIOR, C. M.; UGRINOWITSCH, H.; BENDA, R. N.; CHIVIACOWSKY, S.; CORRÊA, U. C. Pesquisa na área de comportamento motor: modelos teóricos, métodos de investigação, instrumentos de análise, desafios, tendências e perspectivas. Rev. Educ. Fís./UEM, Maringá, v. 21, n. suplemento, p. 1-52, 2010.

THELEN, E.; FISHER, D. M.; RIDLEY-JOHNSON, R. The relationship between physical growth and a newborn reflex. Infant Behavior \& Development, [s. I.], v. 7, n. 4, p. 479-493, 1984.

ULRICH, D. The test of gross motor development. Austin: ProdEd, 2000.

VALENTINI, N. Validity and reliability of the TGMD-2 for Brazilian children. Journal of Motor Behavior, [s. I.], v. 44, n. 4, p. 275-280, 2012.

\section{Publisher}

Universidade Federal de Goiás. Faculdade de Educação Física e Dança. Publicação no Portal de Periódicos UFG. As ideias expressadas neste artigo são de responsabilidade de seus autores, não representando, necessariamente, a opinião dos editores ou da universidade. 